\section{Mutually reinforcing patterning mechanisms}

\section{Elena R. Alvarez-Buylla, Mariana Benítez and Carlos Espinosa-Soto}

In a recent Opinion article published in Nature Reviews Molecular Cell Biology (Computational morphodynamics of plants: integrating development over space and time. Nature Rev. Mol. Cell Biol. 12, 265-273 $(2011))^{1}$, Roeder and collaborators integrate some of the recent work in the emerging field of plant computational morphodynamics, focusing on models based on cellular liveimaging data. One of the examples these authors review is that of Arabidopsis thaliana root epidermal patterning, for which they discuss two mathematical models, namely the WEREWOLF (WER) self-activation model, which we authored ${ }^{2}$, and the mutual support model $^{3}$. They rule out the WER self-activation model, based on experimental data showing that expression of the MYB transcription factor WER is the same in wild-type cells and wer loss-of-function mutant cells. Moreover, Roeder et al. did not consider the results and discussions presented in a more recent version of the WER self-activation model $^{4}$ and in a further theoretical paper ${ }^{5}$.

We argue that the WER self-activation model ${ }^{4}$ - which assumes that WER can positively regulate itself, either directly or through the action of other genes ${ }^{2,4}$ - cannot be ruled out based only on the expression of WER in wer mutants. A similar situation was encountered for the AGAMOUS self-regulatory loop in flower morphogenesis ${ }^{6}$. In the case of A. thaliana root epidermal patterning, recent experimental data show that WER expression is downregulated by the Leu-rich repeat receptor-like kinase SCRAMBLED (SCM $)^{7-9}$ and that $S C M$ expression is, in turn, downregulated by $W E R^{8,9}$, giving rise to a positive feedback loop. This results in the indirect and local self-activation of WER and stabilizes SCM-mediated position signalling. The presence of WER in wer mutants, which was used to discount our model, indeed seems to contradict these data. However, both pieces of evidence can be reconciled if WER is also regulated by other factors, and thus its promoter can still be activated in wer mutants. Furthermore, additional evidence in support of WER being self-activated is that the closely related MYB gene MYB23 — which can substitute for the function of $W E R^{10}$ - participates in a positive feedback loop with itself. This suggests that the observed cell pattern can still occur in wer mutants, owing to the self-maintained expression levels of MYB23 (REF. 10).

This correspondence may be important for the interpretation of experimental data and modelling studies in other cases, given that the coexistence of various apparently redundant circuits seems to be relevant for the robustness of morphogenetic systems ${ }^{5}$. In the particular case of $A$. thaliana epidermal cell patterning, we argue that the WER self-activation model cannot be ruled out on the basis of the evidence currently available, as published data may still support it. We believe that other circuits, such as the mutual support model, are also consistent with the available data and are possibly reinforcing each other, conferring robustness to the overall spatiotemporal cellular patterns ${ }^{5,11}$.

Elena R. Alvarez-Buylla and Mariana Benitez are at the Centro de Ciencias de la Complejidad (C3), Torre de Ingeniería, Ciudad Universitaria, Ciudad de México, Distrito Federal, Mexico.

Elena R. Alvarez-Buylla is also at the Instituto de Ecología, Universidad Nacional Autónoma de México, Ciudad Universitaria 3er Circuito Exterior, Junto Jardín Botánico Exterior, Coyoacán, Ciudad de México, Distrito Federal, Mexico.

Carlos Espinosa-Soto is at the Centro de Investigación y de Estudios Avanzados del Instituto Politécnico Nacional (CINVESTAV-IPN), Edificio Langebio A, Laboratorio 8, Km 9.6 Libramiento Norte Carretera Irapuato-León, 36821 Irapuato, Guanajuato, Mexico.

Correspondence to E.R.A.-B. e-mail:eabuylla@gmail.com doi: 10.1038/nrm3079-c1
1. Roeder, A. H. et al. Computational morphodynamics of plants: integrating development over space and time. Nature Rev. Mol. Cell Biol. 12, 265-273 (2011). 2. Benítez, M., Espinosa-Soto, C., Padilla-Longoria, P., Díaz, J. \& Alvarez-Buylla, E. R. Equivalent genetic regulatory networks in different contexts recover contrasting spatial cell patterns that resemble those in Arabidopsis root and leaf epidermis: a dynamic model. Int. J. Dev. Biol. 51, 139-155 (2007).

Savage, N. S. et al. A mutual support mechanism through intercellular movement of CAPRICE and GLABRA3 can pattern the Arabidopsis root epidermis. PLoS Biol. 6, e235 (2008)

4. Benítez, M., Espinosa-Soto, C., Padilla-Longoria, P. \& Alvarez-Buylla, E. R. Interlinked nonlinear subnetworks underlie the formation of robust cellular patterns in Arabidopsis epidermis: a dynamic spatial model. BMC Syst. Biol. 2, 98 (2008).

5. Benítez, M. \& Alvarez-Buylla, E. R. Dynamic-module redundancy confers robustness to the gene regulatory network involved in hair patterning of Arabidopsis epidermis. Biosystems 102, 11-15 (2010).

6. Alvarez-Buylla, E. R. et al. (2010) Flower development. The Arabidopsis Book 8, e0127 (doi:10.1199/ tab.0127).

7. Schiefelbein, J., Kwak, S. H., Wieckowski, Y., Barron, C. $\&$ Bruex, A. The gene regulatory network for root epidermal cell-type pattern formation in Arabidopsis. J. Exp. Bot. 60, 1515-1521 (2009).

8. Kwak, S. \& Schiefelbein, J. A feedback mechanism controlling SCRAMBLED receptor accumulation and cell-type pattern in Arabidopsis. Curr. Biol. 18, 1949-1954 (2008).

9. Kwak, S. H. \& Schiefelbein, J. Regulated accumulation of the SCRAMBLED receptor and position-dependent cell type patterning in Arabidopsis. Plant Signal. Behav. 4, 332-335 (2009)

10. Kang, Y. H. et al. The MYB23 gene provides a positive feedback loop for cell fate specification in the Arabidopsis root epidermis. Plant Cell 21, 1080-1094 (2009)

11. Benítez, M., Monk, N. \& Alvarez-Buylla, E. R. Epidermal patterning in Arabidopsis: models make a difference. J. Exp. Zool. B Mol. Dev. Evol. 316 , 241-253 (2011).

Competing interests statement

The authors declare no competing financial interests. 